\title{
Ciclo reproductivo de Anadara tuberculosa (Sowerby, 1833) (Arcidae) en Bahía Magdalena, México
}

\author{
Reproductive cycle of Anadara tuberculosa (Sowerby, 1833) \\ (Arcidae) in Magdalena Bay, Mexico
}

\section{Federico A. García-Domínguez ${ }^{1}$, Alejandro De Haro-Hernández², Ángel García-Cuellar, Marcial Villalejo-Fuerte ${ }^{1}$ y Sonia Rodríguez-Astudillo ${ }^{1}$}

\author{
${ }^{1}$ Centro Interdisciplinario de Ciencias Marinas, Av. IPN s/n. Apdo. Postal 592, La Paz 23096, Baja California Sur, México \\ ${ }^{2}$ Centro de Bachillerato Ind. Tecnológico y de Servicios 62. Jalisco s/n La Paz, B.C.S., México \\ ${ }^{3}$ Centro de Bachillerato Ind. Tecnológico y de Servicios 230. 20 de Noviembre y Bordo s/n. La Paz, B.C.S., México \\ fcipactli@hotmail.com
}

\begin{abstract}
The reproductive cycle of Anadara tuberculosa (Sowerby, 1833) and its relationship with temperature and food availability in Magdalena Bay, Mexico was studied. Twenty five adult specimens were collected, on a monthly basis, between January 1998 and February 1999. Gonads were processed by routinary histology. The reproductive cycle was divided in five stages: resting, developing, ripe, spawning and spent. The developing stage was found within the interval March-October and December 1998, and also in February 1999. Spawning was observed within intervals: January-April and June-December 1998, reaching the highest frequencies in: January (60\%), September (60\%), and December (71.4\%). Ripeness occurred within periods: January-March and JuneDecember 1998, reaching the highest frequencies in July (70\%) and August (57.8\%). A significant relationship was found between temperature and ripe stage. The annual mean concentration of chlorophyll $a$ was $2.8 \mathrm{mg} \mathrm{m}^{-3}$, the lowest concentrations were $0.5 \mathrm{mg} \mathrm{m}^{-3}$ (January) and $0.8 \mathrm{mg} \mathrm{m}^{-3}$ (February and December). The highest chlorophyll $a$ rate (9.4 $\mathrm{mg} \mathrm{m}^{-3}$ ) corresponded to May 1998. A significant correlation was found between specimens in developing stage and food availability. It is concluded that the reproductive cycle of $A$. tuberculosa exhibits some characteristics of molluscs living in temperate and subtropical waters, because its reproduction occurs during the whole year, although it has two massive spawning periods.
\end{abstract}

Key words: Bivalvia, mangrove cockle, reproduction, spawning
Resumen.- Se estudió el ciclo reproductivo de Anadara tuberculosa (Sowerby, 1833) y su relación con la temperatura y disponibilidad de alimento en Bahía Magdalena, México. Se recolectaron 25 ejemplares adultos por mes, entre enero de 1998 y febrero de 1999. Las gónadas fueron examinadas mediante histología rutinaria. El ciclo gonadal fue dividido en cinco fases: reposo, desarrollo, madurez, emisión de gametos y post-emisión. La fase de desarrollo se observó en el intervalo marzo-octubre y en diciembre de 1998, así como en febrero de 1999. El desove se observó en los intervalos: enero-abril y junio-diciembre de 1998, con las mayores frecuencias en enero $(60 \%)$, septiembre $(60 \%)$ y diciembre $(71,4 \%)$. La madurez se observó en los intervalos: enero-marzo y junio-diciembre de 1998 , con las mayores frecuencias en julio (70\%) y agosto $(57,8 \%)$. Se encontró una relación significativa entre la temperatura y la fase de madurez. La concentración media anual de clorofila $a$ fue de $2,8 \mathrm{mg} \mathrm{m}^{-3}$, las concentraciones más bajas se observaron en enero $\left(0,5 \mathrm{mg} \mathrm{m}^{-3}\right)$, febrero y diciembre $(0,8$ $\left.\mathrm{mg} \mathrm{m}^{-3}\right)$, y la máxima $\left(9,4 \mathrm{mg} \mathrm{m}^{-3}\right)$ en mayo de 1998 . Se encontró una correlación significativa entre la frecuencia de individuos en fase de desarrollo y la disponibilidad de alimento. Se concluye que el ciclo reproductivo de $A$. tuberculosa presenta características de moluscos que habitan aguas subtropicales y templadas, ya que su reproducción se presenta prácticamente todo el año, aunque con dos periodos masivos de emisión de gametos.

Palabras clave: Bivalvia, pata de mula, reproducción, emisión de gametos

\section{Introducción}

La almeja 'pata de mula' Anadara tuberculosa (Sowerby, 1833), es un bivalvo de manglar explotado en todo el litoral del Pacífico. Es una especie con grandes posibilidades para el desarrollo pesquero (Cruz \& Palacios 1983). En su fase adulta, habita sólo en pantanos de manglares, siempre asociada al mangle rojo Rhizophora mangle en la zona intermareal, donde vive casi completamente enterrada en el fango. Se distribuye desde las costas de Baja California (México) hasta Perú (Poutiers 1995). En Baja California Sur, la zona de pesca tradicional para A. tuberculosa es Bahía Magdalena donde se captura artesanalmente, con una producción media de 673 toneladas anuales de 1991 a 1995 (Hernández-Valenzuela 1996).

Para esta especie, se han realizado algunos estudios del ciclo reproductivo en diversas zonas de México, como los de Flores (1971) en las costas de Sinaloa; Baqueiro 
et al. (1982), en la bahía de La Paz; Pérez-Medina (2005), en Bahía Magdalena. En otros países de Latinoamérica, destacan los trabajos de Squire et al. (1977) y de Borda \& Cruz (2004) en las costas de Colombia y de Cruz-Soto \& Jiménez (1994) en Punta Morales, Costa Rica. Sin embargo, en A. tuberculosa no hay estudios que aborden la relación entre el ciclo reproductivo y la temperatura del medio ambiente, mientras que se han realizado numerosos trabajos en otras especies de bivalvos, como Pinctada mazatlanica (Sevilla 1969, García-Domínguez et al. 1996 y García-Cuéllar et al. 2004), Modiolus capax (Ochoa-Báez 1985), Chione californiensis (GarcíaDomínguez et al. 1993), P. fucata (Behzadi et al. 1997), Spondylus leucacanthus (Villalejo-Fuerte \& GarcíaDomínguez 1998), Paphies donacina (Marsden 1999), Limnoperna fortunei (Darrigan et al. 1999) y Saccostrea palmula (Romo-Piñera 2005).

Por otra parte, una de las etapas críticas de la reproducción es la temporada de desove, que en invertebrados marinos se encuentra relacionada con una alta disponibilidad de alimento (Starr et al. 1990, Jaramillo et al. 1993). También se ha relacionado la disponibilidad de alimento y los valores máximos abundancia de fitoplancton en el medio ambiente con el inicio de la gametogénesis en los bivalvos Pinctada mazatlanica (García-Domínguez et al. 1996), Megapitaria squalida (Villalejo-Fuerte et al. 1996b), Spondylus leucacanthus (Muñetón-Gómez et al. 2001) y S. calcifer (Villalejo-Fuerte et al. 2002).

Bahía Magdalena es una laguna costera de alta productividad de moluscos bivalvos y con un alto impacto pesquero en la economía de México. Sin embargo, se han realizado pocos trabajos sobre la biología reproductiva de las especies objeto de pesquerías, entre las que se cuentan Chione californiensis (GarcíaDomínguez et al. 1993, Prado-Ancona 1998), Argopecten circularis (Félix-Pico et al. 1995), Saccostrea palmula (Romo-Piñera 2005) y Anadara tuberculosa (PérezMedina 2005). El presente trabajo tiene como objetivo analizar la relación entre el ciclo reproductivo de $A$. tuberculosa, la temperatura del medio y la concentración de clorofila $a$ como un indicador del alimento disponible.

\section{Material y métodos}

En Bahía Magdalena (Fig. 1) se muestrearon con periodicidad mensual (enero de 1998 a febrero de 1999) 25 ejemplares de Anadara tuberculosa en el sustrato adyacente a las raíces de los mangles. Los animales fueron transportados al laboratorio y fijados en formol al $10 \%$ en agua de mar.

Para el análisis histológico se obtuvo una porción de

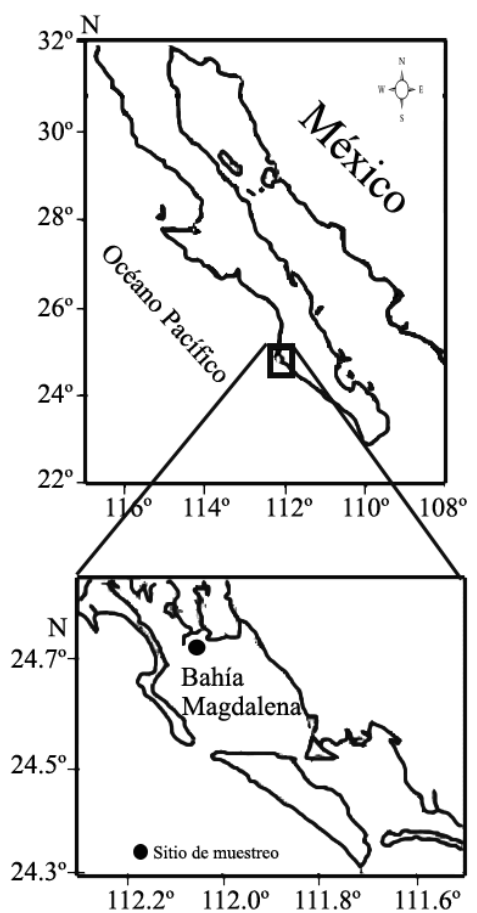

Figura 1

Localización del área de muestreo en Bahía Magdalena, México

Sampling area in Magdalena Bay, Mexico

tejido de la región dorsal, donde está situada la gónada. Las muestras se deshidrataron en una batería creciente de etanol y se incluyeron en parafina. Se realizaron cortes de $7 \mu \mathrm{m}$ de grosor y fueron teñidos con hematoxilinaeosina (Lynch et al. 1972). Finalmente, los cortes se deshidrataron con etanol, se aclararon con xileno y el montaje definitivo se hizo con resina sintética.

Para la caracterización de las diferentes fases de desarrollo de la gónada, se siguió el criterio definido por García-Domínguez et al. (1998) (Tabla 1), que consiste en dividir el ciclo gonadal en cinco fases: reposo, desarrollo, madurez, emisión de gametos y post-emisión de gametos. Cada uno de los ejemplares analizados se clasificó en una de estas fases y se obtuvo la frecuencia relativa mensual de cada una de ellas. Debido a que no se observaron diferencias entre la duración de las fases gonádicas de hembras y machos, se hizo un solo ciclo para ambos sexos (Baqueiro et al. 1982, GarcíaDomínguez et al. 1998, Villalejo-Fuerte et al. 2002).

Con el fin de correlacionar el ciclo reproductivo con las variables ambientales se midió diariamente la temperatura superficial del agua del mar con un 
Tabla 1

Fases de desarrollo de las gónadas de Anadara tuberculosa

Gonadal developmental stages of Anadara tuberculosa

\begin{tabular}{|c|c|c|}
\hline Fase gonádica & Hembras & Machos \\
\hline Reposo & \multicolumn{2}{|c|}{$\begin{array}{l}\text { Presencia de acinos vacios; no se observan gametos. No se puede diferenciar el sexo. El tejido } \\
\text { conjuntivo es abundante y ocupa todo el espacio situado entre el manto, el hepatopáncreas y el tubo } \\
\text { digestivo }\end{array}$} \\
\hline Desarrollo & $\begin{array}{l}\text { Ovocitos dentro de los folículos. } \\
\text { Ovocitos en crecimiento adheridos a la } \\
\text { pared folicular. El centro de algunos } \\
\text { folículos se observa vacio. Disminuye el } \\
\text { área ocupada por el tejido conjuntivo }\end{array}$ & $\begin{array}{l}\text { Cantidad variables de células germinales y } \\
\text { de espermatozoides dentro de los túbulos } \\
\text { seminales. Disminuye el área ocupada por el } \\
\text { tejido conjuntivo. }\end{array}$ \\
\hline Madurez & $\begin{array}{l}\text { Folículos llenos de ovocitos maduros; las } \\
\text { paredes foliculares aún tienen adheridos } \\
\text { algunos ovocitos en crecimiento. } \\
\text { Casi no se observa tejido conjuntivo }\end{array}$ & $\begin{array}{l}\text { Túbulos seminales llenos de espermatozoides. } \\
\text { Se observa una delgada capa de células } \\
\text { espermatogénicas en la pared de los túbulos. } \\
\text { Casi no se observa tejido conjuntivo }\end{array}$ \\
\hline $\begin{array}{l}\text { Emisión de } \\
\text { gametos }\end{array}$ & $\begin{array}{l}\text { Folículos casi vacios, con algunos } \\
\text { ovocitos o vacios por completo. Entre } \\
\text { ellos se observa tejido conjuntivo }\end{array}$ & $\begin{array}{l}\text { Túbulos seminales parcialmente vacios. La } \\
\text { cantidad de espermatozoides presentes } \\
\text { disminuye drásticamente }\end{array}$ \\
\hline $\begin{array}{l}\text { Post-emisión } \\
\text { de gametos }\end{array}$ & $\begin{array}{l}\text { Algunos ovocitos residuales; gran cantidad } \\
\text { de amebocitos que los fagocitan. No se } \\
\text { observa ovogénesis activa }\end{array}$ & $\begin{array}{l}\text { Túbulos seminales colapsados; gran cantidad } \\
\text { de amebocitos fagocitando espermatozoides } \\
\text { residuales. No se observa espermatogénesis } \\
\text { activa }\end{array}$ \\
\hline
\end{tabular}

termómetro de cubeta al $0,01^{\circ} \mathrm{C}$ más próximo. Como temperatura media, se consideró el promedio de todos los datos obtenidos en el mes. Además, en el día del muestreo de organismos, se midió la concentración de clorofila a como indicador de la disponibilidad de alimento. Para ello se tomaron muestras de $2 \mathrm{~L}$ de agua superficial que fueron filtradas a través de filtros Whatman GF/F de fibra de vidrio $(0,07 \mu \mathrm{m}$ de tamaño de poro), y congeladas en nitrógeno líquido. Posteriormente, se hizo una extracción en acetona al $90 \%$ y se analizaron con un espectrofotómetro (Strickland \& Parsons 1972). Este análisis se hizo por duplicado. La clorofila a se calculó utilizando las ecuaciones de Jeffrey \& Humphrey (1975).

Para determinar la relación entre la frecuencia relativa de organismos en cada fase del ciclo reproductivo con la temperatura y con la concentración de clorofila $a$ (se compararon las frecuencias de cada fase, con la media de las temperatura obtenidas durante el mes, y con el dato de concentración de clorofila obtenido el día de la recolecta de almejas), se aplicó un análisis de correlación por rangos de Spearman. Antes de aplicar el análisis de correlación se llevaron a cabo transformaciones logarítmicas para los datos de clorofila $a$ y transformaciones arcoseno para los porcentajes de las fases del ciclo reproductivo con el fin obtener la normalidad y homocedasticidad de los datos (Sokal \& Rohlf 1995). Se informan solo aquellos resultados donde hubo correlación significativa.

\section{Resultados}

\section{Ciclo reproductivo}

La fase de reposo (Fig. 2) se observó durante casi todo el periodo estudiado, excepto en los meses de julio, septiembre y noviembre. La fase de desarrollo (Figs. 3A y 4A), se observó de mayo a diciembre de 1998 y en febrero de 1999, la mayor frecuencia se presentó en junio (85\%). En fase de madurez (Figs. 3B y 4B) se encontraron organismos de enero a marzo y de julio a diciembre de 1998. La emisión de gametos (Figs. 3C y 4C) presentó dos periodos de máxima frecuencia durante 1998, el primero de enero a marzo, en el que se encontró el $50 \%$ de organismos recolectados en esta fase. El segundo periodo fue de septiembre a diciembre del mismo año, registrando en noviembre el máximo porcentaje $(71,4 \%)$. La post-emisión de gametos (Figs. 3D y 4D) se observó de enero a junio y en octubre y noviembre de 1998, y en enero y febrero de 1999. Las frecuencias relativas combinadas para hembras y machos de las fases gonádicas encontradas durante el periodo de estudio se representan en la Fig. 5. 
Vol. 43, Nº 1,2008

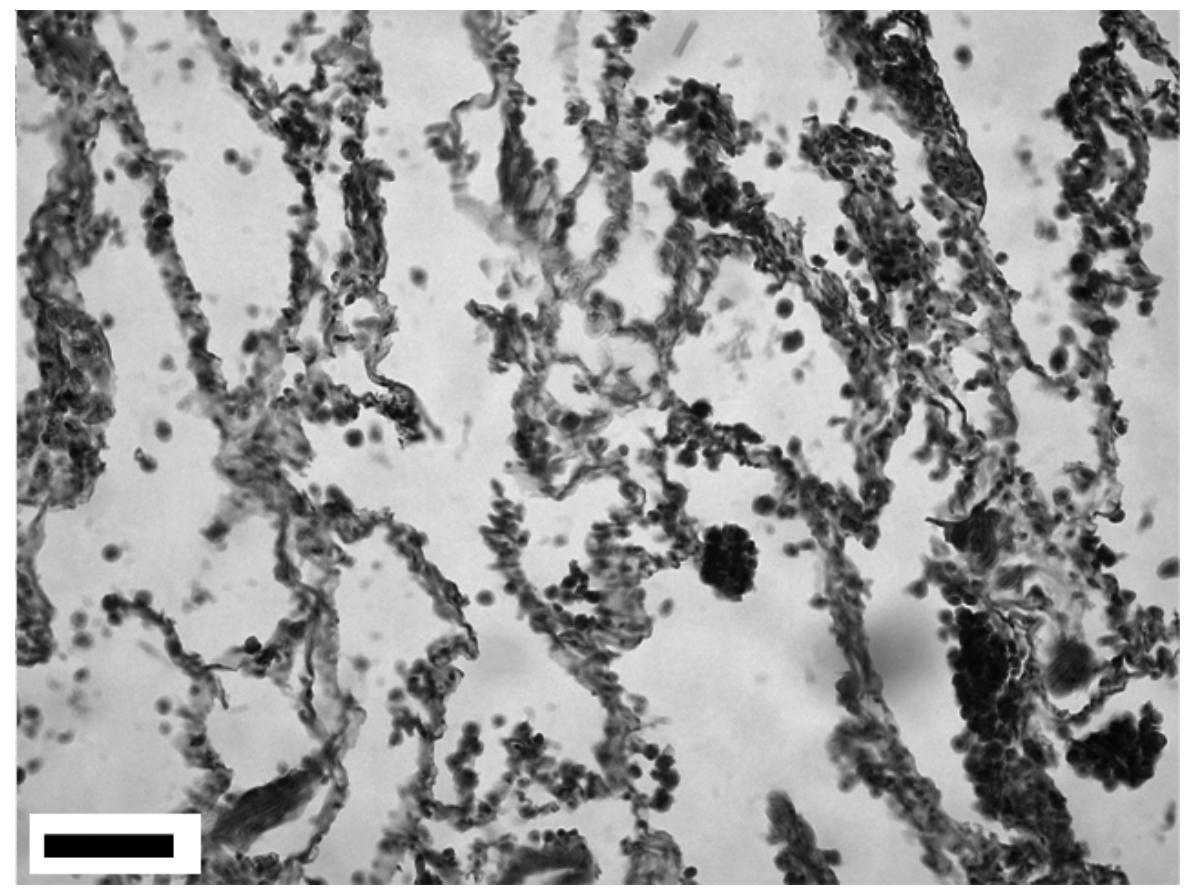

Figura 2

Microfotografía de la gónada en fase de reposo de Anadara tuberculosa. Escala de la barra $=\mathbf{5 0} \boldsymbol{\mu m}$

Photomicrograph of gonadal resting stage in Anadara tuberculosa. Scale bar $=50 \mu \mathrm{m}$

\section{Ciclo reproductivo y disponibilidad de alimento}

La concentración promedio anual de clorofila $a$ fue de $2,8 \mathrm{mg} \mathrm{m}^{-3}$, las concentraciones menores correspondieron a enero $\left(0,5 \mathrm{mg} \mathrm{m}^{-3}\right)$, febrero y diciembre $\left(0,8 \mathrm{mg} \mathrm{m}^{-3}\right)$. La máxima $\left(9,4 \mathrm{mg} \mathrm{m}^{-3}\right)$ se presentó en mayo (Fig. 6).

Se observó una correlación significativa entre la frecuencia de organismos en desarrollo con el aumento en la disponibilidad de alimento $(\mathrm{r}=0,72, P<0,05)$. El incremento en el porcentaje de organismos en esta fase ocurrió después de alcanzar la máxima concentración de clorofila durante 1998.

Las frecuencias más altas de individuos en fase de desove están relacionados inversamente con la concentración de clorofila y el análisis estadístico muestra una correlación significativa $(\mathrm{r}=-0,79, P<0,05)$.

\section{Ciclo reproductivo y temperatura}

La temperatura media en 1998 fue de $22,8^{\circ} \mathrm{C}$. El registro más alto fue de $29,4^{\circ} \mathrm{C}$ y correspondió a agosto (Fig. 6). En diciembre se obtuvo una medición de $18,7^{\circ} \mathrm{C}$, que fue la menor. Se encontró una relación significativa de la temperatura con la fase de madurez gonádica $(\mathrm{r}=0,7063$, $P<0,05)$. En el mes de julio, a $26,1^{\circ} \mathrm{C}$, se observó el $70 \%$ de organismos en fase de madurez. En agosto, a $29,4^{\circ} \mathrm{C}$, la frecuencia relativa fue de $57,89 \%$. El coeficiente de correlación entre la frecuencia relativa de organismos en desove y la temperatura no fue significativo.

\section{Discusión}

\section{Ciclo reproductivo}

En Bahía Magdalena, Anadara tuberculosa presentó la fase de emisión de gametos casi todo el año de 1998 (excepto mayo y junio), con dos emisiones masivas: una de enero a marzo y otra de septiembre a diciembre. En enero y febrero de 1999 no se encontraron organismos en emisión de gametos. Por su parte, Pérez-Medina (2005), en el estero de Santo Domingo (Bahía Magdalena), encontró que el porcentaje máximo de organismos en desove se presentó en mayo $(54,16 \%)$ y diciembre $(57,14 \%)$. Aunque en este estudio no se encontraron organismos en fase de emisión de gametos en mayo y junio, Pérez-Medina (2005) encontró en estos meses, la primera emisión de gametos masiva del año. En consecuencia, se considera que potencialmente al interior de Bahía Magdalena, las larvas de esta especie están presentes todo el año.

Por otro lado, Baqueiro et al. (1982) encontraron en la Bahía de La Paz, en el Golfo de California, México, 

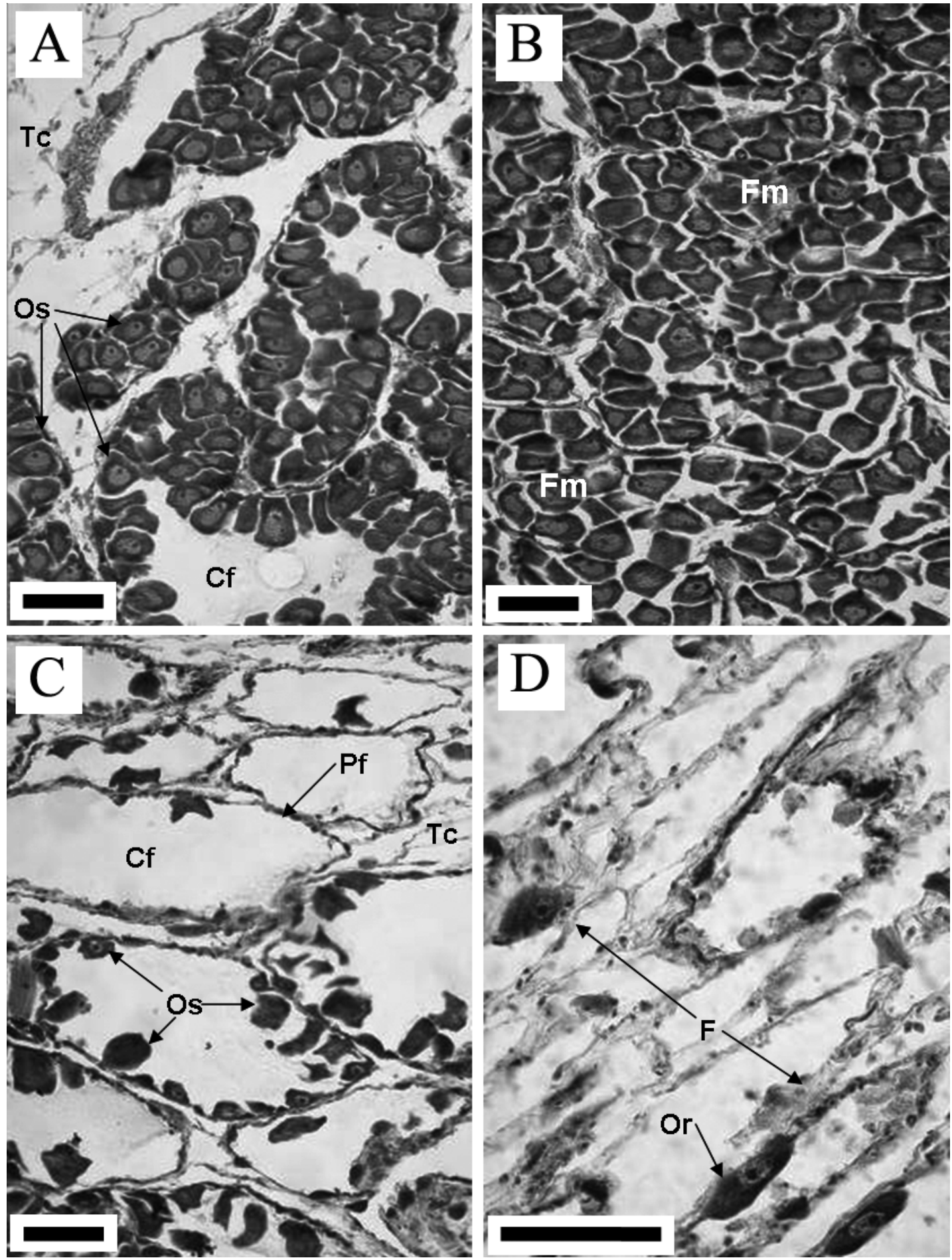

Figura 3

Microfotografías de las fases de la gónada en hembras de Anadara tuberculosa. (A) fase de desarrollo, (B) fase de madurez, (C) fase de emisión de gametos y (D), fase de post-emisión de gametos. Escala de la barra $=50 \mu m$. Tc $=$ tejido conjuntivo, $\mathrm{Cf}=$ centro del folículo, $\mathrm{Os}=$ ovocitos, $\mathrm{Pf}=$ pared folicular, $\mathrm{Or}=\mathrm{Ovocito}$ residual, $\mathrm{Fm}=$ folículo maduro, $\mathrm{F}=$ amibocitos

Photomicrographs of gonadal stages in females of Anadara tuberculosa. (A) developing stage, (B) ripe stage, (C) spawning stage, and (D) spent stage. Scale bar $=50 \mu \mathrm{m} . \mathrm{Tc}=$ connective tissue, $\mathrm{Cf}=$ follicle center, $\mathrm{Os}=$ oocytes, $\mathrm{Pf}=$ folicular wall, $\mathrm{Or}=$ residual oocytes, $\mathrm{Fm}=$ mature follicle, $\mathrm{F}=$ amebocytes 

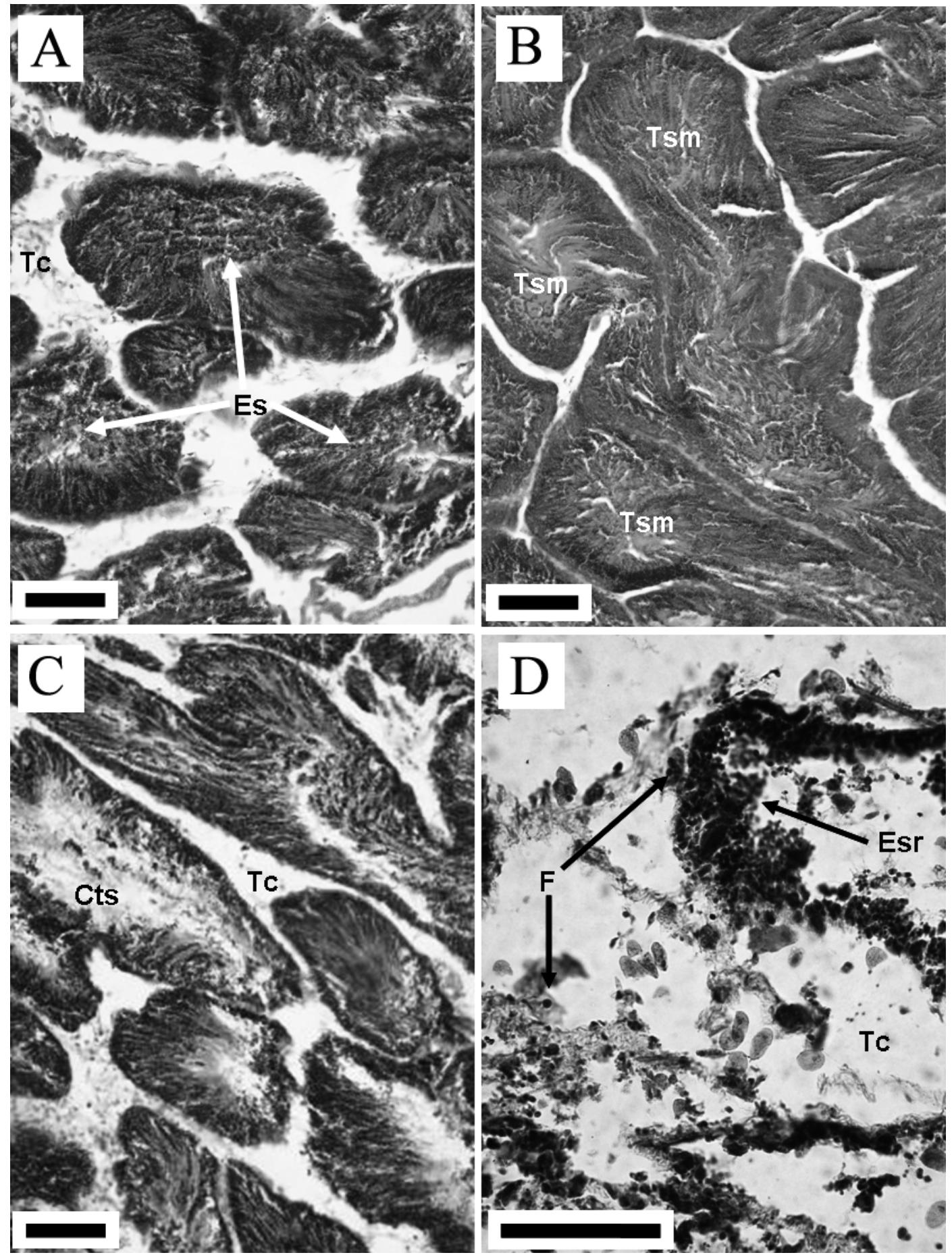

Figura 4

Microfotografías de las fases de la gónada en machos de Anadara tuberculosa. (A) fase de desarrollo, (B) fase de madurez, (C) fase de emisión de gametos y (D), fase de post-emisión de gametos. Escala de la barra $=\mathbf{5 0} \mu \mathrm{m} . \mathrm{Tc}=$ tejido conjuntivo, Es $=$ espermatozoides, $\mathrm{Tsm}=$ túbulo seminal maduro, $\mathrm{Cts}=$ centro del túbulo seminal, $\mathrm{Esr}=$ espermatozoides residuales, $\mathrm{F}=$ amebocitos

Photomicrographs of gonadal stages in males of Anadara tuberculosa. (A) developing stage, (B) ripe stage, (C) spawning stage, and (D) spent stage. Scale bar $=50 \mu \mathrm{m} . \mathrm{Tc}=$ connective tissue, $\mathrm{Es}=$ spermatozoa, $\mathrm{Tsm}=$ mature follicle, $\mathrm{Cts}=$ follicle center, Esr $=$ residual spermatozoa, $\mathrm{F}=$ amebocytes 


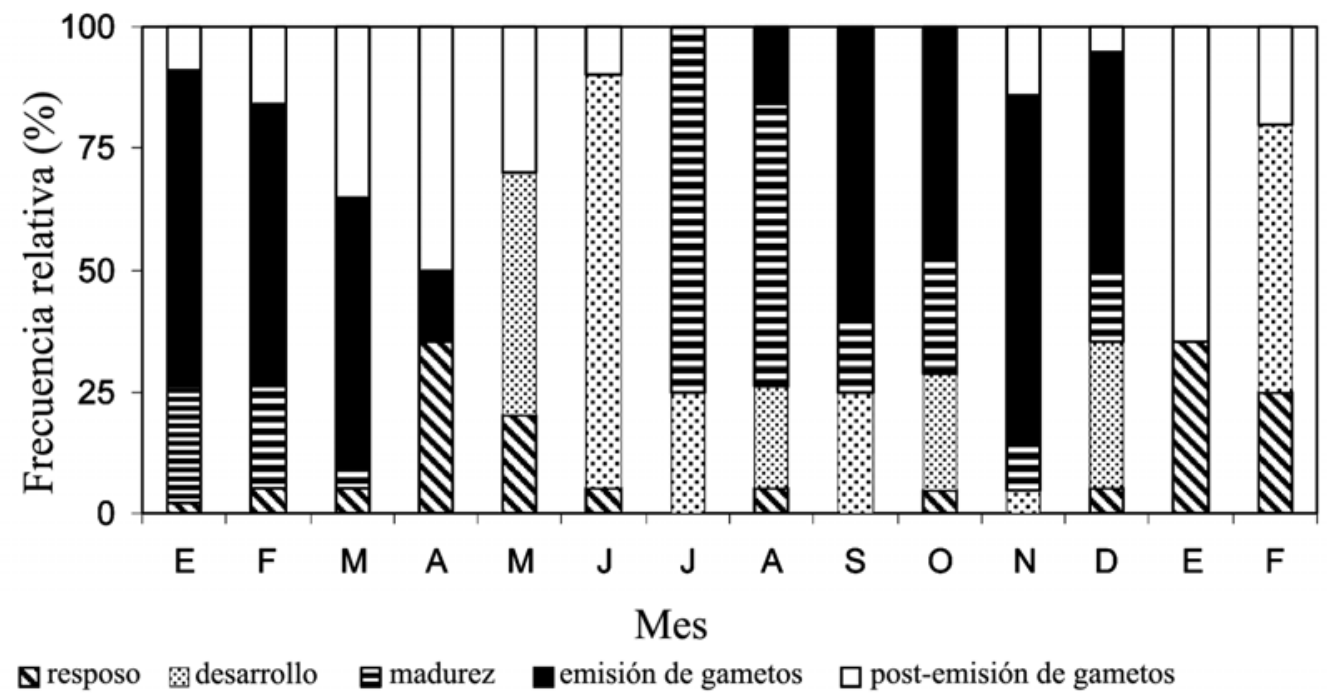

Figura 5

Frecuencias relativas de las fases del ciclo reproductivo de Anadara tuberculosa desde enero 1998 a febrero 1999, en Bahía Magdalena, México

Relative frequencies of the reproductive cycle stages of Anadara tuberculosa from January 1998 to February 1999, in Magdalena Bay, Mexico

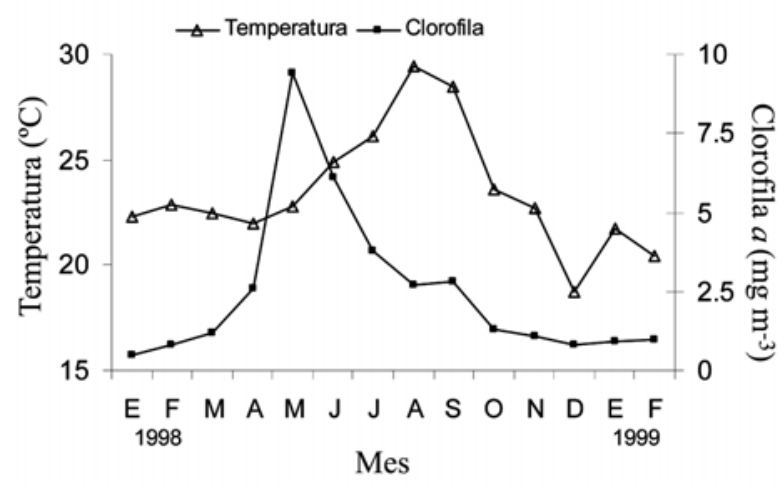

Figura 6

Secuencia de temperaturas del agua de mar y concentraciones medias de clorofila $a$, desde enero 1998 a febrero 1999, en Bahía Magdalena, México

Sequence of sea water temperature and average concentrations of chlorophyll $a$, from January 1998 to February 1999, in Magdalena Bay, Mexico

porcentajes elevados de organismos en fase de desove en abril (95\%) y en agosto (73\%) de 1978 y en enero $(90 \%)$ de 1979. Flores (1971), en un estudio realizado con la misma especie en las costas de Sinaloa, en el Pacífico mexicano, encontró que el periodo de desove se limitaba a los meses de abril a septiembre con un registro máximo en agosto. Ortiz-Ordóñez (1992) plantea que la liberación de gametos masculinos en A. tuberculosa se presenta durante todo el año, pero no presenta porcentajes de frecuencias de ello y su estudio se limitó solo a machos.

En Punta Morales, Costa Rica, Cruz-Soto \& Jiménez (1994) encontraron que el desove de esta especie ocurre entre mayo y septiembre, a diferencia de lo que publicó Cruz (1984), referente a la misma zona, en donde afirma que se encontraron organismos desovando durante todo el año. En Colombia, Borda \& Cruz (2004) también informan que $A$. tuberculosa se reproduce todo el año, pero con una época principal de maduración en noviembre y febrero.

Dadas las diferencias expuestas en el comportamiento temporal del ciclo reproductivo de Anadara tuberculosa en los estudios en referencia y lo observado en el presente trabajo, no es posible establecer una temporalidad reproductiva típica de la especie.

Una posible explicación a las diferencias encontradas en la presente descripción del ciclo reproductivo, de esta especie, respecto a lo encontrado por diversos autores en estudios similares, es que en 1997-1998 se presentó el fenómeno climatológico de El Niño y en 1998-1999 el de La Niña, eventos que afectan a la duración e intensidad del desove de los bivalvos, como lo encontraron GarcíaCuéllar et al. (2004), en la madreperla, Pinctada mazatlanica, especie cuyo desove fue de mayor duración 
e intensidad durante El Niño, y corto y tardío durante La Niña, en la Isla Espíritu Santo, Golfo de California, México. De manera similar, este fenómeno, que en Bahía Magdalena indujo anomalías positivas de temperatura hasta de $+4.4^{\circ} \mathrm{C}$ en verano de 1997 y causó cambios importantes en la abundancia de peces y copépodos (Palomares-García et al. 2003), pudo inducir variaciones en el comportamiento reproductivo de A. tuberculosa en Bahía Magdalena, donde el desove fue más prolongado en 1998 que el encontrado por Pérez-Medina (2005) en el año 2000.

\section{Ciclo reproductivo y disponibilidad de alimento}

El desarrollo de la gónada implica una alta demanda de energía, la cual procede del alimento ingerido directamente del medio ambiente, de productos de reserva o de ambos. Los periodos de mayor disponibilidad de alimento coinciden con el desarrollo gonadal, también la estacionalidad del desarrollo gonadal está vinculada con el almacenamiento y utilización de reservas acumuladas en el organismo en épocas de abundancia (Mackie 1984).

Anadara tuberculosa aparentemente vincula su fase de desarrollo con el alimento que consume directamente del medio, más que con la probable acumulación de reservas, ya que se observó una relación directa y significativa entre la etapa de desarrollo y la época de mayor concentración de clorofila $a$. Romo-Piñera (2005) menciona que en Saccostrea palmula de Bahía Magdalena, la energía obtenida del alimento ingerido se utiliza para el desarrollo de los gametos, lo cual puede ocurrir también en A. tuberculosa. Por su parte, PérezMedina (2005) no encontró una relación clara entre las fases del ciclo gonadal y la concentración de clorofila $a$. Sin embargo, los valores altos del índice de madurez coinciden con las mayores concentraciones de clorofila $a$.

Muchos bivalvos marinos, tienden a reproducirse cuando la disponibilidad de alimento es alta, brindando a la progenie mayores probabilidades de alimentarse y teniendo por otro lado, la posibilidad de recuperar la energía empleada en el desove (Bayne 1965), así la fase de desove coincide con la mayor concentración de fitoplancton, aumentando la probabilidad de que las larvas se alimenten y sobrevivan (Villalejo-Fuerte et al. 1996a).

En Anadara tuberculosa no ocurre lo anterior porque los periodos de desove coinciden con las menores concentraciones de clorofila $a$ (se observó una relación inversa significativa entre esta fase del ciclo gonadal y la disponibilidad de alimento). Esto indica la posibilidad de que el gasto energético necesario para la expulsión de gametos esté sostenido por sustancias energéticas almacenadas, debido a la alta frecuencia de organismos en fase de emisión de gametos y el poco alimento, de manera similar a lo registrado por Muñetón-Gómez et al. (2001) en un estudio sobre Spondylus leucacanthus en isla Danzante, Golfo de California. Además, en el estudio de Pérez-Medina (2005), la máxima frecuencia relativa de organismos en desove se presentó en diciembre, mes en el que registró la concentración más baja de clorofila $a$.

En Bahía Magdalena se encontró que los valores bajos en concentración de clorofila $a$ se registraron para los meses fríos (enero y febrero) y el valor máximo se presentó en mayo (mes cálido). Esto coincide con lo encontrado por Rosales-Villa (2004) que en la misma zona, registró el valor más alto en la concentración de clorofila $a$ en el mismo mes (mayo) y el valor más bajo en febrero. También Pérez-Medina (2005) observó la máxima concentración de clorofila $a$ en mayo y las menores en noviembre y diciembre.

Debido a que el desarrollo gametogénico de $A$. tuberculosa depende del alimento ingerido inmediatamente del medio, mientras que el desove es sostenido probablemente por reservas energéticas almacenadas, es posible concluir que la especie tiene una estrategia reproductiva mixta, entre las especies conservadoras, cuyos ciclos de almacenamiento de nutrientes están claramente separados de los ciclos de producción de gametos y las oportunistas, en las que ambos ciclos se realizan simultáneamente (Bayne 1976, Kang et al. 2000).

\section{Ciclo reproductivo y temperatura}

La temperatura es uno de los factores externos más importantes que influyen en el ciclo reproductivo de los bivalvos. En algunas especies, el desove se inicia cuando la temperatura excede de un nivel crítico característico (Mackie 1984). García-Cuéllar et al. (2004) señalaron que el desove de Pinctada mazatlanica ocurría entre $25^{\circ} \mathrm{C}$ y $27^{\circ} \mathrm{C}$. En Chione californiensis, el desove se presentó cuando la temperatura era mayor de $24^{\circ} \mathrm{C}$ (GarcíaDomínguez et al. 1993) y en Modiolus capax y Spondylus leucacanthus cuando alcanzaba $\operatorname{los} 23^{\circ} \mathrm{C}$ (Ochoa-Báez 1985, Villalejo-Fuerte \& García-Domínguez 1998). El desove de Saccostrea palmula en Bahía Magdalena se inició a partir de los $21,4^{\circ} \mathrm{C}$ (Romo-Piñera 2005).

Según Cruz (1984), la época de desove de $A$. tuberculosa estuvo marcada por altas temperaturas y salinidades del agua. Sin embargo, Baqueiro et al. (1982) y Flores (1971) no observaron con claridad una relación entre altas temperaturas y períodos de desove en dicha especie. Análogamente, no se ha podido establecer una 
relación clara entre la temperatura del agua y el desove de A. tuberculosa en el presente estudio.

Aunque no se observó una relación de este factor ambiental con la emisión de gametos de Anadara tuberculosa, sí se registró una relación significativa con la fase de madurez y el incremento en la temperatura. En contraste, Pérez-Medina (2005) no encontró una relación clara entre la temperatura y las fases del ciclo reproductivo.

En otros bivalvos la temperatura se relaciona con las fases de desarrollo y madurez; Ortega-Hernández \& Arroyo-Hernández (1987) encontraron en Crassostrea virginica, que la etapa de madurez y desove coincidían con valores altos de temperatura. En Chione californiensis, García-Domínguez et al. (1993) encontraron una relación directa entre la fase de gametogénesis y el aumento de la temperatura.

Anadara tuberculosa al presentar actividad de desove todo el año con dos periodos máximos de desove, en Bahía Magdalena, según lo expuesto por Pérez-Medina (2005) y lo observado en este trabajo, se podría afirmar que su comportamiento reproductivo es intermedio entre el presentado por las especies de climas templados y por las de climas tropicales, lo cual es razonable ya que el área de estudio se encuentra en la zona de transición templado-tropical. Es probable también que este comportamiento se deba a las modificaciones ambientales inducidas por El Niño 1997-1998 y La Niña 1998-1999, ya que el periodo de muestreo coincidió con el final del primero y con el inicio del segundo.

\section{Agradecimientos}

Los autores agradecen al Instituto Politécnico Nacional el financiamiento del proyecto que permitió realizar este trabajo. Además desean agradecer profundamente las valiosas observaciones y correcciones efectuadas por los revisores, que indudablemente mejoraron el trabajo y sin las que no hubiera sido posible lograr su publicación. También desean expresar su agradecimiento a la Editora de la Revista y a sus colaboradores por su valiosa ayuda, por su desinteresado apoyo y por su infinita paciencia. F. García-Domínguez, M. Villalejo-Fuerte y S. RodríguezAstudillo, agradecen a la COFAA-IPN, las becas de exclusividad otorgadas.

\section{Literatura citada}

Baqueiro CE, M Muciño \& R Merino. 1982. Análisis de una población de pata de mula Anadara tuberculosa sujeta a explotación intensiva en la Bahía de La Paz, Baja California Sur, México. Ciencia Pesquera 3: 75-82.
Bayne BL. 1965. Growth and the delay of metamorphosis of the larvae of Mytilus edulis (L.). Ophelia 2: 1-47.

Bayne BL. 1976. Aspects of reproduction in bivalve molluscs. En: Wiley M (ed). Estuarine Processes. Vol. 1. Uses, stresses and adaptation to the estuary, pp. 432-448. Academic Press, New York.

Behzadi S, K Parivar \& P Roustain. 1997. Gonadal cycle of pearl oyster, Pinctada fucata (Gould) in Northeast Persian Gulf, Iran. Journal of Shellfish Research 16: 129-135.

Borda CA \& R Cruz. 2004. Reproducción y reclutamiento del molusco Anadara tuberculosa (Sowerby, 1833) en el Pacífico colombiano. Revista Investigaciones Marinas 25: 185-195.

Cruz RA \& J Palacios. 1983. Biometría del molusco Anadara tuberculosa en Punta Morales, Puntarenas, Costa Rica. Revista de Biología Tropical 31: 175-179.

Cruz RA. 1984. Algunos aspectos de la reproducción en Anadara tuberculosa (Pelecypoda: Arcidae) de Punta Morales, Puntarenas, Costa Rica. Revista de Biología Tropical 32: 4550.

Cruz-Soto RA \& JA Jiménez. 1994. Moluscos asociados a las áreas de manglar de la costa pacífica de América Central, 182 pp. Editorial Fundación UNA, Heredia.

Darrigan G, P Penchaszadeh \& MC Damborea. 1999. The reproductive cycle of Limnoperna fortunei_(Dunker, 1857) (Mytilidae) from a neotropical temperate locality. Journal of Shellfish Research 18: 361-365.

Félix-Pico E, MT Ibarra-Cruz, RE Merino-Márquez, VALevyPérez, F García-Domínguez \& R Morales-Hernández. 1995. Reproductive cycle of Argopecten circularis in Magdalena Bay, B.C.S. Mexico. IFREMER, Actes de Colloques 17: 51-155.

Flores MA. 1971. Contribución al conocimiento biológico de la 'Pata de mula' Anadara tuberculosa (Sowerby, 1833) Tesis de Biología, Escuela Nacional de Ciencias Biológicas, Instituto Politécnico Nacional, México DF, 57 pp.

García-Cuéllar JA, F García-Domínguez, D Lluch-Belda \& S Hernández-Vázquez. 2004. El Niño and La Niña effects on reproductive cycle of the pearl oyster Pinctada mazatlanica (Hanley, 1856) (Pteriidae) at Isla Espiritu Santo in the Gulf of California. Journal of Shellfish Research 23: 13-120.

García-Domínguez F, G García-Melgar \& P GonzálezRamírez. 1993. Ciclo reproductivo de la almeja roñosa Chione californiensis (Broderip, 1835) en Bahía Magdalena, B.C.S., Mexico. Ciencias Marinas 19: 5-28.

García-Domínguez F, BP Ceballos-Vázquez \& A TrippQuezada. 1996. Spawning cycle of pearl oyster, Pinctada mazatlanica (Hanley, 1856) (Pteriidae) at Isla Espíritu Santo, B.C.S., México. Journal of Shellfish Research 15: 297-303.

García-Domínguez F, BP Ceballos-Vázquez, M ArellanoMartínez \& M Villalejo-Fuerte. 1998. Reproductive cycle of the giant reef clam Periglypta multicostata (Sowerby, 1835) (Pelecypoda: Veneridae) at Isla Espiritu Santo, Baja California Sur, Mexico. Journal of Shellfish Research 17: 1009-1013.

Hernández-Valenzuela R. 1996. Pesquería de almeja pata de mula. En: Casas-Valdez M \& G Ponce-Díaz (eds), Estudio del Potencial Pesquero y Acuícola de Baja California Sur, pp. 121-126. SEMARNAP, Gobierno de B.C.S., La Paz, México. 
Jaramillo R, J Winter, J Valencia \& A Rivera. 1993. Gametogenic cycle of the Chiloe scallop (Chlamys amandi). Journal of Shellfish Research 12: 59-64.

Jeffrey SW \& GF Humprey. 1975. New spectrophotometric equation for determining chlorophyll's $a, b, c 1$, and $c 2$ in algal phytoplankton and higher plants. Biochemie und Physiologie der Pflanzen 167: 191-194.

Kang CK, MS Park, PY Lee, WJ Chol \& WH Lee. 2000. Seasonal variations in condition, reproductive activity, and biochemical composition of the pacific oyster, Crassostrea gigas (Thunberg), in suspended culture in two coastal bays of Korea. Journal of Shellfish Research 19: 771-778.

Lynch MJ, SS Raphael, LD Mellor, PD Spare \& MJH Inwood. 1972. Métodos de Laboratorio, 1522 pp. Editorial Interamericana, México.

Mackie GL. 1984. Bivalves. En: Tompa AS, NH Verdonk \& J Van Der Biggelaar (eds), The Mollusca: Reproduction 7: 351418. Academic Press, New York.

Marsden I. 1999. Reproductive cycles of the surf beach clam Paphies donacina (Spengler, 1793) from New Zealand. Journal of Shellfish Research 18: 539-546.

Muñetón-Gómez MS, M Villalejo-Fuerte \& I GárateLizárraga. 2001. Contenido estomacal de Spondylus leucacanthus (Bivalvia: Spondylidae) y su relación con la temporada de reproducción y la abundancia de fitoplancton en Isla Danzante, Golfo de California. Revista de Biología Tropical 49: 581-590.

Ochoa-Báez R. 1985. Antecedentes sobre el ciclo de reproducción de Modiolus capax (Conrad, 1873) (Bivalvia: Mytilidae) en la Bahía de La Paz, Baja California Sur, México. Investigaciones Marinas CICIMAR 2: 86-103.

Ortega-Hernández S \& J Arroyo-Hernández. 1987. Observaciones sobre la reproducción de Crassostrea virginica (Gmelin) en la Laguna de Tamiahua, Veracruz. I. Memorias del IX Congreso Nacional de Zoología. Universidad Juárez Autónoma de Tabasco. Villahermosa, Tabasco, México: 105110 .

Ortiz-Ordóñez E. 1992. Histología y ciclo gonádico de ejemplares macho de Anadara (Anadara) tuberculosa (Sowerby, 1833). Tesis de Biología, Escuela Nacional de Ciencias Biológicas, Instituto Politécnico Nacional, México DF, 32 pp.

Palomares-García R, A Martínez-López, R De Silva-Dávila, R Funes-Rodríguez, MA Carvallido-Carranza, $R$ Avendaño-Ibarra, A Hinojosa-Medina \& GA LópezIbarra. 2003. Biological effects of El Niño 1997-1998 on a shallow subtropical ecosystem: Bahia Magdalena, Mexico. Geofísica Internacional 42: 455-466.

Pérez-Medina DR. 2005. Biología reproductiva de Anadara tuberculosa (Bivalvia: Arcidae) en el Estero Santo Domingo, B.C.S., México. Tesis de Maestría, Centro Interdisciplinario de Ciencias Marinas, Instituto Politécnico Nacional, La Paz, México, 70 pp.
Poutiers JM. 1995. Guía FAO para la identificación de especies para los fines de la pesca. Pacifico centro-oriental, Volumen I. Plantas e Invertebrados, Bivalvos, 646 pp. FAO, Roma.

Prado-Ancona JD. 1998. Ciclo reproductivo de tres poblaciones de Chione californiensis (Broderip, 1835) (Bivalvia: Veneridae) en Baja California Sur, México. Tesis de Maestría, Centro Interdisciplinario de Ciencias Marinas, Instituto Politécnico Nacional, La Paz, México, 103 pp.

Romo-Piñera AK. 2005. Ciclo reproductivo del ostión de mangle Saccostrea palmula (Carpenter, 1857), en Bahía Magdalena, Baja California Sur. Tesis de Maestría, Centro Interdisciplinario de Ciencias Marinas, Instituto Politécnico Nacional, La Paz, México, 74 pp.

Rosales-Villa AR. 2004. Dinámica de nutrimentos en Bahía Magdalena, B.C.S. Tesis de Maestría, Centro Interdisciplinario de Ciencias Marinas, Instituto Politécnico Nacional, La Paz, México, 104 pp.

Sevilla ML. 1969. Contribución al conocimiento de la madreperla Pinctada mazatlanica (Hanley 1945). Revista de la Sociedad Mexicana de Historia Natural 30: 223-262.

Sokal RR \& FJ Rohlf. 1995. Biometría. Principios y métodos estadísticos en la investigación biológica, 832 pp. H. Blume Ediciones, Madrid.

Squire HJ, M Estevez, O Barona \& O Mora. 1977. Mangrove cockles, Anadara spp., of the Pacific coast of Colombia. The Veliger 18: 57-68.

Starr M, JH Himmelman \& JC Therriault. 1990. Direct coupling of marine invertebrates spawning with phytoplankton blooms. Science 247(4946): 1071-1074.

Strickland JDH \& TR Parsons. 1972. A practical handbook of seawater analysis, Bulletin of the Fisheries Research Board of Canada 167: 1-310.

Villalejo-Fuerte M, BP Ceballos-Vázquez \& F GarcíaDomínguez. 1996a. Reproductive cycle of Laevicardium elatum, (Sowerby, 1883) (Bivalvia: Cardiidae) in Bahia Concepcion, Baja California Sur, Mexico. Journal of Shellfish Research 15: 741-745.

Villalejo-Fuerte M, G García-Melgar, R Ochoa-Báez \& A García-Gasca. 1996b. Ciclo reproductivo de Megapitaria squalida, (Sowerby, 1835) (Bivalvia: Veneridae) en Bahía Concepción, Baja California Sur, México. Boletín Científico 4: 29-39.

Villalejo-Fuerte M \& F García-Domínguez. 1998. Reproductive cycle of Spondylus leucacanthus Broderip, 1833 (Bivalvia: Spondylidae) at Isla Danzante, Gulf of California. Journal of Shellfish Research 17: 1037-1042.

Villalejo-Fuerte M, M Arellano-Martínez, BP CeballosVázquez \& F García-Domínguez. 2002. Reproductive cycle of Spondylus calcifer Carpenter, 1857 (Bivalvia: Spondylidae) in the 'Bahía Loreto' National Park, Gulf of California, Mexico. Journal of Shellfish Research 21: 103-108. 